\title{
Association of Copeptin, a Surrogate Marker of Arginine Vasopressin, with Decreased Kidney Function in Sugarcane Workers in Guatemala
}

\author{
Jaime Butler-Dawson ${ }^{a-c}$ Miranda Dally ${ }^{a-c}$ Richard J. Johnson $^{c, d}$ Evan C. Johnson ${ }^{e}$ \\ Lyndsay Krisher $^{a, c}$ Laura-Gabriela Sánchez-Lozada ${ }^{f}$ Benjamin R. Griffin ${ }^{d}$ \\ Stephen Brindley ${ }^{b}$ Lee S. Newman ${ }^{a-c, g, h}$ \\ ${ }^{a}$ Center for Health, Work, and Environment, Colorado School of Public Health, University of Colorado, \\ Aurora, CO, USA; ${ }^{b}$ Department of Environmental and Occupational Health, Colorado School of Public Health, University \\ of Colorado, Aurora, CO, USA; 'C Colorado Consortium on Climate Change and Human Health, University of Colorado, \\ Aurora, CO, USA; ${ }^{\mathrm{d}}$ Division of Renal Diseases and Hypertension, Department of Medicine, School of Medicine, University \\ of Colorado, Aurora, CO, USA; ${ }^{~}$ Division of Kinesiology and Health, University of Wyoming, Laramie, WY, USA; ${ }^{f}$ Department \\ of Cardio-Renal Physiopathology, Instituto Nacional de Cardiología-Ignacio Chávez, Mexico City, Mexico; ${ }^{9}$ Division \\ of Pulmonary Sciences and Critical Care Medicine, Department of Medicine, School of Medicine, Aurora, CO, USA; \\ ${ }^{\mathrm{h}}$ Department of Epidemiology, Colorado School of Public Health, University of Colorado, Aurora, CO, USA
}

\section{Keywords}

Vasopressin · Kidney · Hydration · Agricultural workers

\begin{abstract}
Background: Vasopressin is elevated in response to heat and dehydration and has been postulated to have a role in the chronic kidney disease of unknown origin being observed in Central America. The aims of this study were to examine whether the vasopressin pathway, as measured by copeptin, is associated with the presence of kidney dysfunction, and to examine whether higher fluid intake is associated with lower circulating copeptin and thereby preserves kidney health among sugarcane workers exposed to hot conditions. Methods: Utilizing a longitudinal study of 105 workers in Guatemala, we examined relationships between hydration indices, plasma copeptin concentrations, and kidney function markers at 3 times during the 6-month harvest. We also examined whether baseline copeptin concentrations increased the odds of developing an estimated glomerular filtration rate (eGFR) $<60 \mathrm{~mL} / \mathrm{min} / 1.73 \mathrm{~m}^{2}$. Results:
\end{abstract}

(C) 2020 S. Karger AG, Basel

www.karger.com/anm

Karger ${ }^{\prime}=$
Copeptin concentrations were positively associated with serum creatinine $(\beta 1.41,95 \% \mathrm{Cl} 0.88-2.03)$ and negatively associated with eGFR $(\beta-1.07,95 \% \mathrm{Cl}-1.43$ to -0.70$)$. In addition, as workers improved their hydration (measured by increases in fluid balance), copeptin concentrations were reduced, and this reduction was associated with an improvement in kidney function. Conclusions: Results suggest that copeptin should be studied as a potential prognostic biomarker.

(c) 2020 S. Karger AG, Basel

\section{Introduction}

A high prevalence of chronic kidney disease of unknown origin $(\mathrm{CKDu})$ has been observed among agricultural populations in the Americas and Asia [1]. The pathophysiology of CKDu is unknown. However, agricultural workers perform intense physical work in high ambient temperatures and humidity, which can result in dehydration and potentially produce stress on the kidney [2]. This has led to the 
hypothesis that chronic exposure to heat stress with concomitant dehydration may increase individual risk and to the need to investigate underlying mechanisms [3].

One potential candidate for inducing renal injury by heat stress is vasopressin. While vasopressin can help the host through urinary concentration, recurrent and chronically elevated vasopressin has been shown to produce experimental renal injury, in part by inducing oxidative stress as well as activation of the polyol pathway [4]. Because vasopressin is unstable with a short half-life, copeptin, a stable component of pre-pro-vasopressin and marker of circulating vasopressin, has emerged as a surrogate biomarker [5] and may help explain biological response mechanisms induced by dehydration and other vasopressin-related stimuli. For example, higher circulating copeptin levels have been associated with kidney dysfunction in general populations [6] and microalbuminuria [7]. Furthermore, there is increasing experimental evidence that chronic elevations in vasopressin may lead to glomerular and tubular damage [8].

We have performed a longitudinal study among sugarcane workers in southwest Guatemala where we evaluated the relationship of plasma copeptin concentrations to hydration and renal function at several times during a harvest season. Our hypothesis was that vasopressin levels (measured via copeptin) are associated with impaired renal function in a sugarcane worker population. Copeptin may help explain biological response mechanisms induced by dehydration and other vasopressin-related stimuli.

Our first aim was to examine the relationships between repeated measures of plasma copeptin concentrations and markers of kidney function and injury including serum creatinine, estimated glomerular filtration rate (eGFR), urine neutrophil gelatinase-associated lipocalin, and urine albumin-to-creatinine ratio. Our second aim was to examine the association between increased fluid balance and decreases in copeptin concentrations and, in turn, associations with improvements in kidney function. Our third aim was to evaluate the merits of copeptin as an early stage clinical biomarker in agricultural workers at risk for kidney dysfunction, by testing whether higher copeptin concentrations at study baseline were associated with increased odds of having an eGFR $<60 \mathrm{~mL} /$ $\mathrm{min} / 1.73 \mathrm{~m}^{2}$ at the end of the study.

\section{Methods}

\section{Setting and Participants}

This research was conducted in a prospective cohort of male sugarcane field workers in Guatemala in 2016-17. The study is de- scribed in detail elsewhere $[9,10]$. A schematic of the study timeline is provided in the online supplement Figure S1 (for all online suppl. material, see www.karger.com/doi/10.1159/000506619). Survey data and urine and blood samples were collected at the end of the work shift for 105 workers at 3 time points in February, March, and April 2017 [9]. All participants provided written consent prior to study enrollment. The study was approved by the Institutional Review Board of the University of Colorado and in Guatemala (Comite de Etica, Facultad de Medicina, Universidad Francisco Marroquin-Hospital Universitario Esperanza).

\section{Data Collection and Laboratory Analysis}

Details on urine and blood measurements and covariate data collection are in the supplement. Copeptin concentration was measured in plasma-EDTA samples and analyzed using an immunoassay analyzer (Kryptor Compact Plus, Thermo Scientific B.R.A.H.M.S Copeptin proAVP, Hennigsdorf, Germany) with a limit of detection of $0.69 \mathrm{pmol} / \mathrm{L}$.

\section{Statistical Analysis}

Pearson correlation coefficients were used to assess the relationships between plasma copeptin concentrations and hydration indices. Normality was visually assessed and met by the Central Limit Theorem. We also examined relationships between other potential covariates that may be correlated with copeptin, including creatine kinase and tons of cane cut per day [11], electrolyte solution intake [12], and sugar-sweetened beverage intake [13].

To evaluate our first aim, whether repeat cross-sectional measures of plasma copeptin concentrations were associated with markers of kidney function and injury, we used linear mixed-effect models with a random intercept for each study subject, estimating $\beta$ and $95 \%$ CIs. We controlled for potential confounders a priori, including hemoglobin Alc (HbAlc), age, systolic and diastolic blood pressure, and change in bodyweight (as an indirect measure of fluid retention) [14]. To assess our second aim, whether increased fluid balance was associated with decreases in plasma copeptin concentrations and, in turn, associated with improvements in kidney function, we computed longitudinal variation in hydration indices (urine specific gravity, urine sodium, serum sodium, and serum osmolality), copeptin and eGFR for each subject between 2 consecutive time points. We then used linear mixed-effect models with a random intercept for each study subject to examine relationships between change in hydration indices, change in copeptin concentration, and change in eGFR while controlling for age, $\mathrm{HbAlc}$, and systolic and diastolic blood pressure. Finally, to address our third aim, we examined the influence of plasma copeptin at study baseline on the odds of having an eGFR $<60 \mathrm{~mL} /$ $\mathrm{min} / 1.73 \mathrm{~m}^{2}$ at the final time point using logistic regression. All analyses were completed in SAS version 9.4 (Cary, NC, USA).

\section{Results}

There were 105 workers who participated in the study with a participation rate of $97 \%$. Participant demographics and clinical characteristics are displayed in Table 1. Copeptin concentration distributions are shown in the online supplement Figure S2. Copeptin concentrations 
Table 1. Characteristics of study participants $(n=105)$

\begin{tabular}{|c|c|}
\hline Characteristics & Mean (SD) \\
\hline Age, years & $30(9)$ \\
\hline \multicolumn{2}{|l|}{ Job, $n(\%)$} \\
\hline Cane cutter & $83(79)$ \\
\hline Cane seeder & $22(21)$ \\
\hline Body mass index, $\mathrm{kg} / \mathrm{m}^{2}$ & $23.48(2.58)$ \\
\hline Systolic BP, mm Hg & $112(10)$ \\
\hline Diastolic BP, mm Hg & $70(9)$ \\
\hline Hypertension $^{\mathrm{a}}, n(\%)$ & $4(4)$ \\
\hline Blood glucose, mg/dL & $81.79(13.69)$ \\
\hline $\mathrm{HbA1c}$ & $5.66(0.29)$ \\
\hline Diabetic, $>6.5 \%, n(\%)$ & $0(0)$ \\
\hline Pre-employment eGFR ${ }^{\mathrm{b}}, \mathrm{mL} / \mathrm{min} / 1.73 \mathrm{~m}^{2}$ & $111.11(15.60)$ \\
\hline $\begin{array}{l}\text { a Defined as systolic } \mathrm{BP} \geq 140 \mathrm{~mm} \\
\geq 90 \mathrm{~mm} \mathrm{Hg} \text {. } \\
\text { b eGFR measured prior to the start of th } \\
\text { BP, blood pressure; eGFR, estimated glc } \\
\text { HbAlc, hemoglobin Alc. }\end{array}$ & $\begin{array}{l}\text { d/or diastolic BP } \\
\text { month harvest. } \\
\text { ular filtration rate; }\end{array}$ \\
\hline
\end{tabular}

averaged $5.79 \pm 6.96 \mathrm{pmol} / \mathrm{L}$ in February, $5.28 \pm 4.67$ in March, and $3.24 \pm 1.70$ in April.

Hydration indices, work practices, and kidney function markers are described in Table 2. On average, most participants produced urine samples consistent with adequate hydration, supported by their positive weight change across the work shift, low urine specific gravity, and normal serum osmolality. Copeptin concentrations were significantly correlated with all hydration indices, except cross-shift weight change, and with several kidney markers.

\section{Aim 1 Results}

Univariate results examining the relationships between serial measures of plasma copeptin concentrations and markers of kidney function are shown in the online supplement Table S1. In the multivariable models, copeptin was positively associated with serum creatinine ( $\beta 1.41,95 \%$ CI $0.88-2.03)$ and inversely related to eGFR $(\beta-1.07,95 \%$ CI -1.43 to -0.70$)$, Table 3. Copeptin was not related to urine neutrophil gelatinase-associated lipocalin or urine albumin-to-creatinine ratio. Of note, $\mathrm{HbA1c}$ was inversely associated with eGFR ( $\beta-15.59$, $95 \%$ CI -26.40 to -4.78 ).

\section{Aim 2 Results}

We constructed 3 models to examine whether increased fluid balance was positively associated with decreases in copeptin concentrations and, in turn, improvements in kidney function between 2 subsequent time points (online suppl. Table S2). In our first model, we observed that increases in urine specific gravity, serum osmolality, and serum sodium were all positively associated with an increase in copeptin concentration, with urine-specific gravity associated with the largest change in copeptin ( $\beta$ 2.68; 95\% CI 1.41-3.94). In the second model, we observed that an increase in copeptin concentration was negatively associated with a decrease in eGFR ( $\beta-1.08 ; 95 \%$ CI -1.62 to -0.54$)$. In our third model, we examined whether change in hydration indices and/or change in copeptin concentration was associated with change in eGFR. We found that change in copeptin concentration was significantly associated with change in eGFR and none of the hydration indices were significant (Models 3a-d, online suppl. Table S2). In the supplementary material, we present a scatter plot that shows changes in urine specific gravity, copeptin, and eGFR in 3 dimensions (online suppl. Fig. S3).

\section{Aim 3 Results}

Our third aim was to evaluate whether higher copeptin concentrations at study baseline were associated with increased odds of having a final eGFR $<60 \mathrm{~mL} / \mathrm{min} / 1.73 \mathrm{~m}^{2}$. The OR of having an eGFR $<60 \mathrm{~mL} / \mathrm{min} / 1.73 \mathrm{~m}^{2}$ was 1.14 (95\% CI $1.05-1.23, p<0.01)$ per $1 \mathrm{pmol} / \mathrm{L}$ increase of copeptin at study baseline. Five workers had an eGFR $<60 \mathrm{~mL} /$ $\mathrm{min} / 1.73 \mathrm{~m}^{2}$ at their last time point (April) and 3 of these workers had high initial copeptin concentrations (range 24-39 pmol/L).

\section{Discussion}

The primary findings of this study were that (1) a reduction of urine concentration (through higher fluid intake) was associated with a reduced concentration of copeptin, a surrogate marker of vasopressin; and (2) a reduction of copeptin concentration was associated with an improvement in markers of kidney function. Our findings indicate that plasma copeptin concentrations may be predictive of kidney function decline over several months. An additional novel finding is that higher plasma copeptin concentrations were associated with worse kidney function as indicated by serum creatinine and eGFR, at multiple time points.

These findings suggest that plasma copeptin concentration may be an early marker of decreased kidney function occurring in sugarcane workers. Experimental studies suggest vasopressin may have a role in the kidney injury in models of CKD and diabetes [15]. Vasopressin
Butler-Dawson et al. 
Table 2. Descriptive data of biomarkers, work practices, and kidney function, averaged from all time points and their correlations with copeptin

\begin{tabular}{|c|c|c|c|c|}
\hline Characteristics & Number & Mean (SD) & \multicolumn{2}{|c|}{ Plasma copeptin } \\
\hline Urine specific gravity & 265 & $1.005(0.007)$ & 0.58 & $<0.01$ \\
\hline$>1.020, n(\%)$ & 265 & $24(9)$ & & \\
\hline Serum osmolality, mmol/kg & 264 & $280.07(7.78)$ & 0.40 & $<0.01$ \\
\hline Creatine kinase, $\mathrm{U} / \mathrm{L}$ & 264 & $403.80(291.76)$ & -0.01 & 0.82 \\
\hline Cane cut during shift, tons & $202^{\mathrm{a}}$ & $6.12(2.26)$ & -0.09 & 0.21 \\
\hline Water, L & 263 & $13.27(5.33)$ & -0.33 & $<0.01$ \\
\hline Electrolyte fluid, $\mathrm{L}^{\mathrm{b}}$ & 260 & $2.50(1.50)$ & -0.32 & $<\mathbf{0 . 0 1}$ \\
\hline Number of sugar-sweetened beverages & 257 & $0.45(0.93)$ & 0.03 & 0.60 \\
\hline Urine ACR, mg/mmol & 252 & $2.58(4.66)$ & -0.01 & 0.86 \\
\hline Copeptin, pmol/L & & $4.73(4.49)$ & $12.37(11.02)$ & $<0.01$ \\
\hline
\end{tabular}

a Reduced $\mathrm{N}$ since $20 \%$ of the participants are seeders and do not cut cane.

${ }^{\mathrm{b}}$ Electrolyte fluid was distributed in 500 -mL bags.

${ }^{\mathrm{c}}$ Conversion factor for creatinine in $\mu \mathrm{mol} / \mathrm{L}$ to $\mathrm{mg} / \mathrm{dL}$ divide by $88.4,{ }^{*} p$ value based on Wilcoxon rank sums test.

ACR, albumin-to-creatinine ratio; NGAL, neutrophil gelatinase-associated lipocalin; eGFR, estimated glomerular filtration rate. Bolded values represent significance at a $p$ value $<0.05$.

Table 3. Results from multivariable linear mixed-effect models examining associations between plasma copeptin and kidney function markers

\begin{tabular}{|c|c|c|c|c|c|c|c|c|}
\hline & \multicolumn{2}{|c|}{ Serum creatinine $(n=245)$} & \multicolumn{2}{|l|}{$\operatorname{eGFR}(n=245)$} & \multicolumn{2}{|l|}{ Urine NGAL $(n=234)$} & \multicolumn{2}{|l|}{ Urine ACR $(n=233)$} \\
\hline & estimate $(95 \% \mathrm{CI})$ & $p$ value & estimate (95\% CI) & $p$ value & estimate $(95 \% \mathrm{CI})$ & $p$ value & estimate $(95 \% \mathrm{CI})$ & $p$ value \\
\hline Copeptin & $1.41(0.88$ to 2.03$)$ & $<0.01$ & $-1.07(-1.43$ to -0.70$)$ & $<0.01$ & $-0.04(-0.23$ to 0.15$)$ & 0.70 & $-0.004(-0.87$ to 0.86$)$ & 0.99 \\
\hline HbAlc & $19.28(6.73$ to 31.84$)$ & $<0.01$ & $-15.59(-26.40$ to -4.78$)$ & $<0.01$ & $3.43(-3.64$ to 10.50$)$ & 0.34 & $0.64(-1.63$ to 2.91$)$ & 0.58 \\
\hline Age, years & $0.43(0.01$ to 0.84$)$ & 0.04 & $-1.13(-1.46$ to -0.80$)$ & $<0.01$ & $0.06(-0.16$ to 0.28$)$ & 0.60 & $-0.05(-0.11$ to 0.01$)$ & 0.08 \\
\hline Systolic BP & $0.16(-0.36$ to 0.67$)$ & 0.56 & $-0.14(-0.53$ to 0.26$)$ & 0.50 & $0.0004(-0.18$ to 0.18$)$ & 0.99 & $0.01(-0.05$ to 0.08$)$ & 0.69 \\
\hline Diastolic BP & $-0.04(-0.78$ to 0.71$)$ & 0.92 & $-0.12(-0.66$ to 0.42$)$ & 0.66 & $-0.21(-0.45$ to 0.03$)$ & 0.09 & $-0.10(-0.20$ to 0.00$)$ & 0.05 \\
\hline Weight change, $\mathrm{kg}$ & $0.009(-0.42$ to 0.44$)$ & 0.97 & $-0.14(-0.53$ to 0.26$)$ & 0.50 & $0.29(0.01$ to 0.57$)$ & 0.04 & $0.02(-0.12$ to 0.16$)$ & 0.83 \\
\hline
\end{tabular}

eGFR, estimated glomerular filtration rate; NGAL, neutrophil gelatinase-associated lipocalin; ACR, albumin-to-creatinine ratio; BP, blood pressure; HbA1c, hemoglobin A1c. Bolded values represent significance at a $p$ value $<0.05$.

may act in part by increasing glomerular hydrostatic pressure, and vasopressin has been shown to increases albumin excretion in humans [16]. Experimental studies of heat stress-induced kidney injury have also provided evidence that exogenous desmopressin can induce further kidney damage [17], while blocking vasopressin action via the V1a and V2 receptors may ameliorate renal injury [4], especially in the setting where the hydration fluids contain sugar-based solutions containing fructose. Potential mechanisms of injury include elevation in oxidative stress and the concurrent over-activation of the intra-renal polyol (aldose reductase-fructokinase) pathway [4]. The proposed mechanism for these effects is that vasopressin may increase glomerular pressure, through the activation of the polyol pathway and the induction of oxidative stress, leading to tubular and glomerular injury [18]. 
Our findings suggest that copeptin may prove to be a useful clinical and research tool for determining if workers are at risk for kidney dysfunction. One clinical trial observed that increased water intake resulted in an attenuation of copeptin over 6 weeks [19]. However, studies that have included interventions to modify vasopressin secretion to observe changes in kidney outcomes are very limited. One intervention study in stage $3 \mathrm{CKD}$ patients used coaching to increase water intake and observed reductions in copeptin. However, the increased water intake did not show a significant slowing in the decline of kidney function after 1 year [20]. The intervention study was conducted in patients with stage $3 \mathrm{CKD}$ and patients were excluded from the study if they drank $>10$ cups of fluid a day, which is contrary to this current study. Our study participants were drinking on average $13 \mathrm{~L}$ of water per day.

One of the more provocative findings of this study was that the copeptin relationship to eGFR was observed in subjects who were relatively well hydrated. Besides emphasizing the potential importance of vasopressin in mediating kidney disease, this study also provides insights regarding osmolar versus non-osmolar stimuli for vasopressin in the setting of heat stress. By following workplace protocols that strongly encouraged water and electrolyte consumption, some individuals developed mild, asymptomatic hyponatremia and positive weight change across the work shift. The observation that mild hyponatremia developed may indicate that copeptin levels may be higher than expected for the given water intake. Vasopressin can be stimulated by a number of other factors among agricultural workers, including extracellular volume depletion [21] and non-osmolar stimuli (i.e., increased body core temperature, physical exercise, and consumption of fructose in rehydration beverages) [4, 11-13]. However, the relationship of urine sodium concentration with copeptin concentration was opposite of what was expected (as extracellular volume depletion is associated with a low urine sodium concentration and, in this study, copeptin concentration increased with increasing urine sodium concentration). While we observed a negative correlation between electrolyte fluid and copeptin, we did not observe any relationship between intake of sugar-sweetened beverages and copeptin. These beverages have less salt and potassium than electrolyte fluid which could result in less water retention [22] and, thus, result in less attenuation of copeptin. In contrast, a recent study observed greater increases in serum copeptin in healthy adults $(n=12)$ who only drank soft drinks during and after $4 \mathrm{~h}$ of exercise in the heat compared to adults who only drank water [23]. The authors suggest the potential role of vasopressin, uric acid, and the polyol-fructokinase pathway in the development of acute kidney injury when drinking soft drinks and exercising in the heat. We included survey questions regarding consumption of energy drinks, juice, and soda in our study, whereas the soft drink study had the participants only drink Mountain Dew ${ }^{\circledR}$ (Mountain Dew, PepsiCo, Purchase, NY, USA), which has higher fructose contents compared to other sugar-sweetened drinks.

Another interesting result that will warrant further study in populations at risk of $\mathrm{CKDu}$ was that $\mathrm{HbA} 1 \mathrm{c}$ was strongly associated with declines in eGFR. Hyperglycemia and diabetes mellitus are risk factors for kidney dysfunction. However, because of the high prevalence of anemia in Guatemala [24] and because anemia can confound the clinical interpretation of $\mathrm{HbAlc}$ [25], we speculate that HbAlc may, alternatively, imply a relationship between anemia and eGFR in our population.

A strength of this study was that it followed a group of workers, sampled 3 times over 3-month. Despite the inherent challenges of conducting field research under extreme field conditions, the study participation rate was high, and we were able to obtain, transport, and analyze specimens with a high degree of reliability.

There are several study limitations that should be noted. We used plasma copeptin concentration as a surrogate for vasopressin secretion. While a number of studies have validated copeptin as a reliable measure for vasopressin [5], one study found a significant correlation between copeptin and vasopressin levels but copeptin explained only half of the variance of vasopressin [26]. Authors suggest that there is a sharper decrease in the clearance rate of copeptin than vasopressin when GFR decreases. We do not know if the changes in renal function associated with copeptin represent acute, subacute, or chronic changes. We relied on creatinine and eGFR to assess short-term kidney function changes and not actual measured GFR. Actual GFR changes may be even more pronounced than suggested by eGFR changes. There is also the possibility that associations between copeptin and markers of kidney function are unrelated to the pathogenesis of disease. Copeptin may be a functional marker of kidney injury or copeptin may be demonstrating a hemodilution effect, which might be the reason it correlates with eGFR. Nevertheless, the experimental studies showing that chronic vasopressin effects can induce renal damage [4] support the relationship of higher copeptin with reduced eGFR. Serum creatinine was measured separately for each time point, which may lead to anomalous creatinine estimates. For Aim 3, since only 5 participants had an eGFR $<60 \mathrm{~mL} /$ 
$\min / 1.73 \mathrm{~m}^{2}$, we were not able to run a multivariable model, as this would result in overfitting the models. Finally, we relied on self-reported fluid intake. To mitigate the risk of recall bias, we asked participants to recall that day's fluid intake at the end of the work shift.

In summary, our study provides preliminary evidence that copeptin may be an early marker of kidney dysfunction in agricultural workers at high risk for heat stress. Potential clinical and workplace interventions that mitigate this form of injury should be examined in the face of increasing heat extremes. Further research is warranted to understand the contribution of vasopressin to renal injury.

\section{Acknowledgement}

We thank Thermo Fisher Scientific for the support with the Kryptor Compact Plus instrument. We thank all of the workers who have made this work possible.

\section{Statement of Ethics}

All participants provided written consent prior to study enrollment. The study was approved by the Institutional Review Board of the University of Colorado and in Guatemala by the Comite de Etica, Facultad de Medicina, Universidad Francisco MarroquinHospital Universitario Esperanza.

\section{Disclosure Statement}

The University of Colorado has a memorandum of agreement with Pantaleon, a Guatemala-based agribusiness. Pantaleon provides partial financial support for research through a contract with the university and has provided access to the employees who volunteered to participate in this research project. The University of Colorado employed appropriate research methods in keeping with academic freedom, based conclusions on critical analysis of the evidence, and reported findings fully and objectively. The terms of this arrangement have been reviewed and approved by the University of Colorado in accordance with its conflict of interest policies.

\section{Funding Sources}

This study was supported by Centers for Disease Control and Prevention (U19 OH011227) and National Institutes of Health (R21 ES028826), and in part by Pantaleon and the Chancellor, University of Colorado, CU Anschutz Campus. Funders had no role in data analysis, interpretation of data, writing the manuscript, or the decision to submit the findings for publication.

\section{Author Contributions}

J.B.-D., L.K., R.J.J., and L.S.N.: research idea and study design. J.B.-D., M.D., S.B., E.C.J., R.J.J., B.R.G., and L.-G.S.-L.: data analysis/interpretation. J.B.D. and M.D.: statistical analysis. R.J. and L.S.N.: supervision or mentorship.

\section{References}

1 Johnson RJ, Wesseling C, Newman LS. Chronic Kidney Disease of Unknown Cause in Agricultural Communities. N Engl J Med. 2019 May;380(19):1843-52.

2 Nerbass FB, Pecoits-Filho R, Clark WF, Sontrop JM, McIntyre CW, Moist L. Occupational Heat Stress and Kidney Health: From Farms to Factories. Kidney Int Rep. 2017 Aug; 2(6):998-1008.

3 Roncal-Jimenez C, García-Trabanino R, Barregard L, Lanaspa MA, Wesseling C, Harra T, et al. Heat Stress Nephropathy From Exercise-Induced Uric Acid Crystalluria: A Perspective on Mesoamerican Nephropathy. Am J Kidney Dis. 2016 Jan;67(1):20-30.

4 García-Arroyo FE, Tapia E, Blas-Marron MG, Gonzaga G, Silverio O, Cristóbal M, et al. Vasopressin Mediates the Renal Damage Induced by Limited Fructose Rehydration in Recurrently Dehydrated Rats. Int J Biol Sci. 2017 Jul;13(8):961-75.

5 Balanescu S, Kopp P, Gaskill MB, Morgenthaler NG, Schindler C, Rutishauser J. Correlation of plasma copeptin and vasopressin concentrations in hypo-, iso-, and hyperosmolar States. J Clin Endocrinol Metab. 2011 Apr;96(4):1046-52.
6 El Boustany R, Tasevska I, Meijer E, Kieneker LM, Enhörning S, Lefèvre G, et al. Plasma copeptin and chronic kidney disease risk in 3 European cohorts from the general population. JCI Insight. 2018 Jul;3(13):121479.

7 Meijer E, Bakker SJ, Halbesma N, de Jong PE, Struck J, Gansevoort RT. Copeptin, a surrogate marker of vasopressin, is associated with microalbuminuria in a large population cohort. Kidney Int. 2010 Jan;77(1):29-36.

8 Bouby N, Bachmann S, Bichet D, Bankir L. Effect of water intake on the progression of chronic renal failure in the $5 / 6$ nephrectomized rat. Am J Physiol. 1990 Apr;258(4 Pt 2): F973-9.

9 Sorensen CJ, Butler-Dawson J, Dally M, Krisher L, Griffin BR, Johnson RJ, et al. Risk Factors and Mechanisms Underlying Cross-Shift Decline in Kidney Function in Guatemalan Sugarcane Workers. J Occup Environ Med. 2019 Mar;61(3):239-50.

10 Butler-Dawson J, Krisher L, Yoder H, Dally M, Sorensen C, Johnson RJ, et al. Evaluation of heat stress and cumulative incidence of acute kidney injury in sugarcane workers in Guatemala. Int Arch Occup Environ Health. 2019 Oct;92(7):977-90.
11 Kim J, Lee J, Kim S, Ryu HY, Cha KS, Sung DJ. Exercise-induced rhabdomyolysis mechanisms and prevention: A literature review. J Sport Health Sci. 2016 Sep;5(3):324-33.

12 Hew-Butler T. Arginine vasopressin, fluid balance and exercise: is exercise-associated hyponatraemia a disorder of arginine vasopressin secretion? Sports Med. 2010 Jun;40(6):459-79.

13 García-Arroyo FE, Cristóbal M, ArellanoBuendía AS, Osorio H, Tapia E, Soto V, et al. Rehydration with soft drink-like beverages exacerbates dehydration and worsens dehydration-associated renal injury. Am J Physiol Regul Integr Comp Physiol. 2016 Jul;311(1): R57-65.

14 Tasevska I, Enhörning S, Burri P, Melander O. High salt intake increases copeptin but salt sensitivity is associated with fluid induced reduction of copeptin in women. Int J Hypertens. 2014;2014:641587.

15 Bardoux P, Martin H, Ahloulay M, Schmitt F, Bouby N, Trinh-Trang-Tan MM, et al. Vasopressin contributes to hyperfiltration, albuminuria, and renal hypertrophy in diabetes mellitus: study in vasopressin-deficient Brattleboro rats. Proc Natl Acad Sci USA. 1999 Aug;96(18):10397-402. 
16 Bardoux P, Bichet DG, Martin H, Gallois Y, Marre M, Arthus MF, et al. Vasopressin increases urinary albumin excretion in rats and humans: involvement of $\mathrm{V} 2$ receptors and the renin-angiotensin system. Nephrol Dial Transplant. 2003 Mar;18(3):497-506.

17 Roncal-Jimenez CA, Milagres T, Andres-Hernando A, Kuwabara M, Jensen T, Song Z, et al. Effects of exogenous desmopressin on a model of heat stress nephropathy in mice. Am J Physiol Renal Physiol. 2017 Mar;312(3):F418-26.

18 Roncal-Jimenez C, Lanaspa MA, Jensen T, Sanchez-Lozada LG, Johnson RJ. Mechanisms by Which Dehydration May Lead to Chronic Kidney Disease. Ann Nutr Metab. 2015;66 Suppl 3:10-3.

19 Lemetais G, Melander O, Vecchio M, Bottin $\mathrm{JH}$, Enhörning S, Perrier ET. Effect of increased water intake on plasma copeptin in healthy adults. Eur J Nutr. 2018 Aug;57(5):1883-90.

20 Clark WF, Sontrop JM, Huang SH, Gallo K, Moist L, House AA, et al. Effect of Coaching to Increase Water Intake on Kidney Function Decline in Adults With Chronic Kidney Disease: The CKD WIT Randomized Clinical Trial. JAMA. 2018 May;319(18):1870-9.

21 Danziger J, Hoenig MP. The Role of the Kidney in Disorders of Volume: core Curriculum 2016. Am J Kidney Dis. 2016 Nov;68(5): 808-16.

22 Shirreffs SM, Armstrong LE, Cheuvront SN. Fluid and electrolyte needs for preparation and recovery from training and competition. J Sports Sci. 2004 Jan;22(1):57-63.

23 Chapman CL, Johnson BD, Sackett JR, Parker MD, Schlader ZJ. Soft drink consumption during and following exercise in the heat elevates biomarkers of acute kidney injury. Am J Physiol Regul Integr Comp Physiol. 2019 Mar; 316(3):R189-98

24 Pan American Health Organization. Anemia in Latin America and the Caribbean, 2009: Situation analysis, trends and implications for public health programming. Washington (D.C.): PAHO; 2010

25 Rhea JM, Molinaro R. Pathology consultation on $\mathrm{HbA}(1 \mathrm{c})$ methods and interferences. Am J Clin Pathol. 2014 Jan;141(1):5-16.

26 Roussel R, Fezeu L, Marre M, Velho G, Fumeron $\mathrm{F}$, Jungers $\mathrm{P}$, et al. Comparison between copeptin and vasopressin in a population from the community and in people with chronic kidney disease. J Clin Endocrinol Metab. 2014 Dec;99(12):4656-63. 